\title{
Evidence for Surgical Management of Facial Pain and Headache
}

\author{
Shannon Clark MD; Chengyuan Wu MD, MSBmE \\ Department of Neurological Surgery, Thomas Jefferson University, Philadelphia, PA
}

Headache and facial pain are a commonly encountered wide spectrum of complex medical conditions. Unfortunately, aside from treating trigeminal neuralgias, interest in surgical management of facial pain and headache from the neurosurgical community has been historically low. The reasons for this are multifactorial and include waning reimbursement, lack of evidence to support a number of pain procedures, and the absence of pain education in neurosurgical residency programs. In this article, we present surgical therapies currently available for headache and facial pain and review the published evidence for commonly performed neurosurgical treatments for craniofacial pains.

\section{TRIGEMINAL NEURALGIA}

Trigeminal neuralgia (TN) is one of the many types of facial pain syndromes, which has a good evidence-based data for the benefit of surgical management. It is also one of the common conditions treated with microvascular decompression (MVD), internal neurolysis (IN), radiofrequency (RF) rhizotomy, glycerol rhizotomy, and gamma knife radiosurgery (GKRS). TN is thought to occur as a result of compression of the root entry zone of the nerve by the neighboring offending artery igniting the hyper-excitable axons at the trigeminal root. ${ }^{1,2}$ In contemporary neurosurgery, the first line of management for patients suffering from this debilitating disease is medical treatment with carbamazepine or gabapentin. In cases of failed medical therapy or drug intolerance, or simply when patients do not prefer to take these medications for a long period of time, surgical options should be considered. Neurosurgical management of TN include three modalities: craniotomy for MVD or IN, percutaneous techniques, and GKRS. Percutaneous techniques can be further divided into glycerol rhizotomy, balloon compression, and radiofrequency rhizotomy.

\section{A. Microvascular decompression or internal neurolysis for Trigeminal Neuralgia}

The MVD procedure has proven to be an effective and durable treatment with initial pain relief as high as $98 \%$ (of which $82 \%$ was complete relief) and $68 \%$ excellent or good relief at 10 year follow-up..$^{3,4}$ Establishing accurate diagnosis of TN is the key to having a successful outcome for MVD. Miller et $\mathrm{l}^{5}$ reported that patients presenting with Type 1 TN (see table 1 for description) pain ( $\geq 50 \%$ episodic pain), according to the classification of Burchiel, ${ }^{1}$ had significantly higher chance of having a favorable outcome following MVD for TN than patients with type II TN pain (84\% vs $64 \%$ ). One must also keep in mind the possibility of multiple sclerosis induced neuralgia and post-herpetic neuralgia before proceeding with MVD as these are unlikely to respond.

There is a tendency to offer percutaneous procedures or GKRS rather than MVD to patients with advanced age. However, recent studies have shown no significant differences in complications or short-term and long-term outcomes of MVD between elderly patients and in younger ones. ${ }^{6,7}$ One study concluded that although complications show a tendency to increase with advanced age, age itself does not act as a risk factor. ${ }^{8}$ Preoperative identification of an obvious offending vessel on imaging studies and intraoperative identification of an offending artery are also considered as the most significant factors for a favorable outcome. $^{5,9}$ However, a positive MRI finding of a close anatomic relationship or contact between the vessels and the trigeminal nerve is quite commonly reported in patients without $\mathrm{TN}$ and vice versa, patients with classic TN commonly have no such image findings especially with regular MRI or MRA of brain. It is reported that 3D constructive interference in steady state MR, which is heavily T2-weighted sequence with very high resolution of the CSF tissue contrast and high-resolution 3D time-of-flight MRA may provide adequate preoperative information in most cases. ${ }^{10}$ In up to $75 \%$ of cases, the offending vessel is the superior cerebellar artery. Pure venous compression is seen in approximately $12 \%$ and small arteries in 15\%.3,11 Multi-vessel compression consisting of an artery and a vein is reported in as many as $56 \%$ of the patients. ${ }^{11}$ In about $15 \%$ of the patients undergoing MDV, despite satisfactory exploration, no offending vessel can be identified. ${ }^{12}$ For the TNs without neurovascular compression (NVC), internal neurolysis (IN) or "nerve combing" maybe performed. The IN procedure entails separating the nerve longitudinally, using a blunt-tip dissector. The nerve is divided into 8-10 bundles from the pons to the petrous bone. In their long-term follow up of patients treated with IN, Burchiel et al ${ }^{9}$ reported $85 \%$ pain free rate immediately after IN and pain free survival rate of $58 \%$ and $47 \%$ at 1 year and 5 years respectively, which are better than both RF or GKRS

\section{B. Percutaneous treatment for trigeminal neuralgia}

Percutaneous treatment modalities for TN consist of glycerol rhizotomy (GR), radiofrequency thermocoagulation (RT), and balloon compression (BC). All 3 of these treatments are generally safe, efficient, and effective, and rely on the principle of inducing pain relief by direct injury to the trigeminal nerve. We offer GR and RT here in Jefferson. Percutaneous GR is an 
Table 1. Burchiel's classification scheme for facial pains commonly encountered in neurosurgical practice

\begin{tabular}{|l|l|l|}
\hline Pain catergory & History/Pain pattern & Other names \\
\hline Trigeminal neuralgia type 1 & Spontaneous onset $(>50 \%$ episodic pain) & Idiopathic trigeminal neuralgia \\
\hline Trigeminal neuralgia type 2 & Spontanoues onset $(>50 \%$ constant pain) & Atypical trigeminal neuralgia \\
\hline Trigeminal neuropathic pain & Trimeminal injury-unintentional (trauma, sinus surgery) & \\
\hline Trigeminal deafferentation pain & Deafferetation (after destructive procedures) & Anesthesia dolorosa \\
\hline Symptomatic trigeminal neuralgia & Multiple sclerosis & \\
\hline
\end{tabular}

overall safe procedure performed under monitored anesthesia care with low risk of significant morbidity. For this reason, patients with high surgical risk identified by preoperative cardiology evaluation are recommended for GR rather than MVD. The most common postoperative finding is a disturbance of facial sensation that typically lasts for a few hours to 1 or 2 weeks. Complications include facial hypesthesia lasting longer than 2 weeks and the rates range from $0-30 \% .{ }^{13,14}$ Serious complications such as cranial neuropathies and bacterial meningitis are low $(0-2 \%)$ with inadvertent entry into the oral cavity being the most common etiology for bacterial meningitis after GR. ${ }^{15}$

According to Pollock et $\mathrm{al}^{16}$ predictive factors for successful GR included patients without any constant facial pain, patients with immediate facial pain during glycerol injection, and patients with new trigeminal deficits after percutaneous GR. Perhaps the most significant benefit of GR is maintained efficacy and safety with repeat procedure. Bender and colleagues ${ }^{17}$ and Harries and colleagues ${ }^{18}$ described their experience with 100 or more patients with repeat GR and reported similar rates of initial and log-term pain relief to the initial GR without changes in the durability of procedure or increase in morbidity and especially no cases of anesthesia dolorosa. In some institutions, GR is chosen for multiple sclerosis related TN patients with reported $74 \%$ initial pain relief and median pain-free interval of 28 months. ${ }^{19}$

On the other hand, RT or radiofrequency ablation is carried out by thermal lesioning induced by an electrode advanced into the foramen ovale. This procedure requires stimulation mapping to identify optimal locations for lesioning by inducing parastesias in the same pattern as the TN, hence requiring corporation of an awake patient during the procedure. Lesions are performed at a temperature of $60-80^{\circ} \mathrm{C}$ for 30 to 120 seconds. ${ }^{19}$ After hypalgesia has been achieved, the motor function of the trigeminal nerve is also tested. The rates of initial pain relief have been reported as high as $97 \%$ while rates of long-term relief range from $25 \%$ to $95 \%$ but defining long-term recurrence rates becomes difficult because of the variable duration of follow-up. ${ }^{19-21}$ Kanpolat and colleagues ${ }^{21}$ in 2001 reported on their experience with 1600 patients and described $52.3 \%$ of pain relief at 10 year follow-up and $41 \%$ pain relief at 20 -year follow-up. Taha et al ${ }^{22}$ in 1995 described a $25 \%$ recurrence rate after 14 years among their 154 patients. The rate of recurrence correlated with the degree of sensory deficits elicited during the procedure: the more mild the hypalgesia noted, the higher the recurrence rate.

Complications of RT include persistent sensory deficit and paresthesia as a result of the lesioning and the rates range from $0.9 \%$ to $9 \%$ among the large patient series. ${ }^{23,24}$ Corneal anesthesia has been reported to range from 0 to $17 \% .{ }^{19}$ Other complications reported include transient trigeminal motor paresis, anesthesia dolorosa, and very rare CSF rhinorrhea. In an attempt to decrease the rate of complications, $\mathrm{Xu}$ et $\mathrm{al}^{25}$ described their experience with 54 patients using computed tomography neuronavigation and frameless stereotactic cannulation of the foramen ovale. The rate of success increased to $85 \%$ vs $54 \%$ at 12 months. In some institutions, ${ }^{19} \mathrm{RT}$ is preferentially performed for multiple sclerosis-related TN with $86 \%$ immediate pain relief albeit short median time to recurrence (5 months).

\section{GKRS for TN}

In Jefferson, the fourth option for TN is GKRS, with its main advantage being its noninvasiveness and low morbidity rate. The major limitation of radiosurgery as compared with MVD is the slow response time (requires up to 3 months) and limited durability of pain relief. Optimal populations for radiosurgery include patients older than 70 years, patients with multiple sclerosis and patients with significant medical comorbidities. The first prospective study was performed by a group from Marseille, France and was a quality-of-life assessment showing improvement in all quality of life parameters and finding that 58 of $83(70 \%)$ responders were able to come off of medications. ${ }^{26} \mathrm{~A}$ second study carried out at the Mayo Clinic looked at the cost effectiveness of SRS Vs. MVD and found that MVD was more expensive in the near term; but for patients with longer life expectancies, it seemed to be the more cost-effective option. ${ }^{27}$

\section{Comparison of surgical modalities for TN}

An extensive review by Tatli et $\mathrm{al}^{28}$ looked at the outcomes of various surgical modalities published with minimum 5 years of follow-up. Their findings 
suggested that MVD provided the highest rate of long-term patient satisfaction and lowest rate of pain recurrence; among the percutaneous techniques compared with MVD, glycerol rhizotomy had a low initial pain relief and a high pain recurrence rate; balloon compression had a high rate of facial hypoesthesia, and a higher rate of postoperative trigeminal motor dysfunction; GKRS showed a low initial pain relief and a lower pain-free rate in the followup period compared with MVD.

\section{SURGICAL OPTIONS FOR COMPLEX CRANIOFACIAL PAIN}

Craniofacial pain is a common condition that affects approximately $10 \%$ to $25 \%$ of the adult populations with a significant impact on their quality-of-life. The International Headache Society classified craniofacial pain into 14 different categories, ${ }^{29}$ with three large groups: the primary headaches (migraine, cluster $\mathrm{HA}$, etc) the secondary headaches (due to trauma, infection, vascular disorder, substance withdrawal etc) and cranial neuralgias (TN, occipital neuralgias etc). The detailed categories and pathophysiology of each craniofacial pain syndromes are out of the scope of this article. Here, we focus on the data of neurosurgical treatments of these disease entities.

Various modes of surgical intervention with complex headache and face pain include (1) peripheral neuromodulation (peripheral nerve stimulation or ganglion stimulation), (2) spinal cord stimulation, (3) trigeminal tractotomy and caudalis DREZ ablation, and (4) motor cortex stimulation

\section{A. Peripheral neuromodulation}

This treatment modality for neuropathic pain was first introduced by Wall and Sweet in 1967, where 8 patients with intense cutaneous pain, 4 experienced relief for more than half an hour following stimulation of infraorbital and mandibular nerves. ${ }^{30}$ However, it was not until the reintroduction of this modality by Weiner and Reed in 199931 that this therapy gained wider clinical acceptance, specifically for occipital neuralgia. Since then, cranial peripheral nerve stimulation such as occipital and supraorbital nerve stimulations have been utilized as

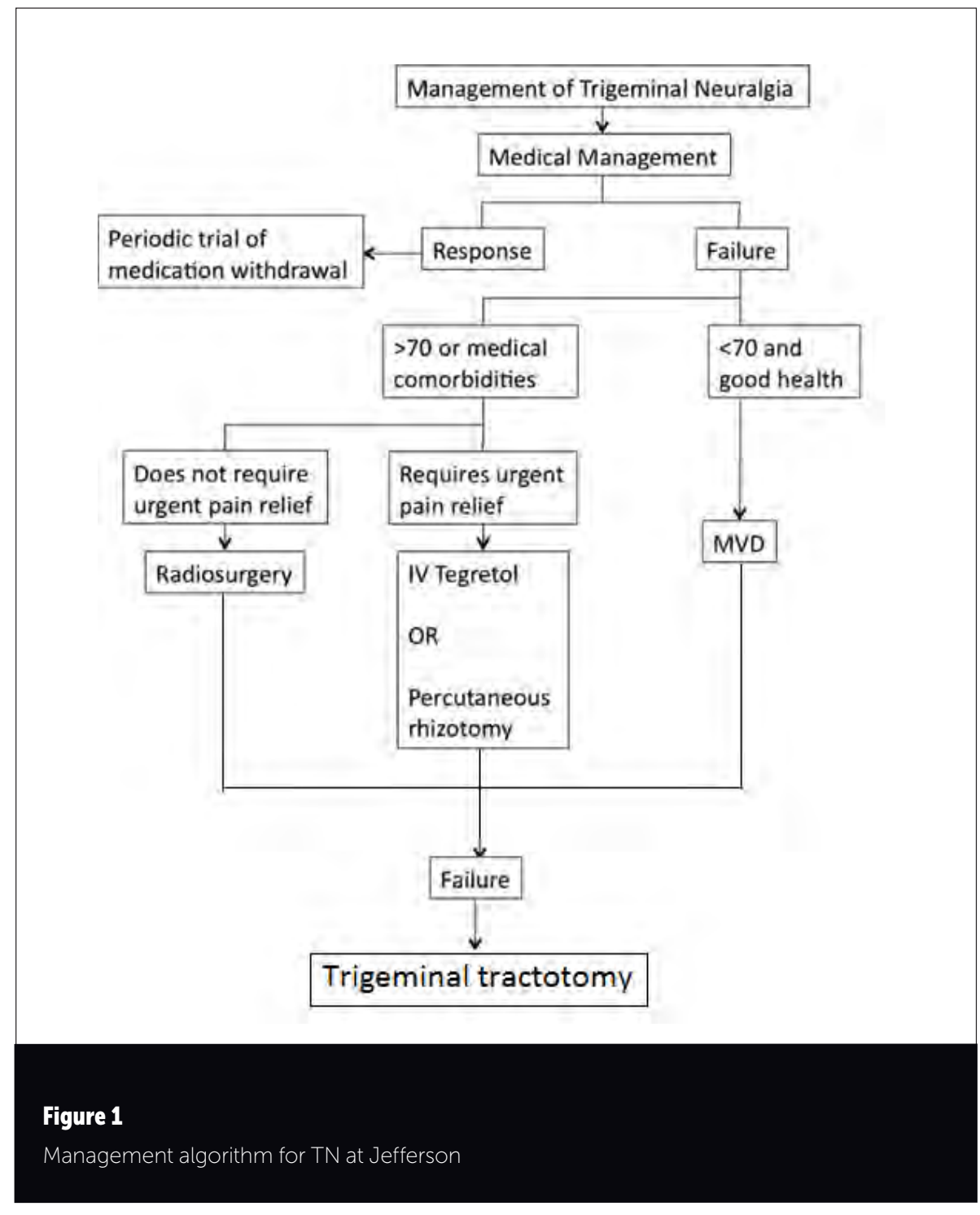

"off-label" treatment for medically refractory migraine, cluster headache, as well as complex craniofacial pain experienced in the trigeminal nerve tributaries. At Jefferson, we perform approximately 5 cases of PNS for such indications each month.

The results of peripheral nerve stimulation (PNS) depend on appropriate patient selection. The prerequisites for this therapy are the following: ${ }^{32,33}$

1. Patients with severe, chronic refractory neuropathic pain that is affecting the patient's quality of life. In addition, nonsurgical options should be exhausted before consideration.
2. There should be some preservation of sensation in the distribution of pain because functioning vibrotactile receptors are mandatory for this therapy to be successful.

3. The pain should be either in the distribution of a single nerve or should be able to be covered by the length of available electrodes for PNS to be successful.

4. Patients should be devoid of underlying psychiatric disorders or secondary gains to their chronic pain disorder

5. A successful trial is mandatory before permanent placement and a 
improvement of greater than $50 \%$ of pain on a visual analog scale (VAS) is generally considered a successful trial.

Despite the longevity of the therapy, few well designed prospective studies of PNS exist (Table 2). A handful of prospective multicenter controlled studies of PNS have been completed. One of the challenges in controlled trials is the lack of blinding, because active stimulation is always associated with paresthesia. In some study designs, low-amplitude and high-amplitude stimulation substituted for the usual sham and active groups to address this limitation. Most of the published literature are single center case series and among these PNS has reported significant improvement ( $>50 \%$ on VAS) in localized chronic pain intensity. Wound breakdown and hardware related issues are the primary complications seen in these procedures. ${ }^{34}$

\section{B. Sphenopalatine ganglion stimu- lation for headache and facial pain}

Electric stimulation of sphenopalatine ganglion (SPG) has recently been shown to be effective in relieving cluster headache pain and the associated autonomic synptoms. ${ }^{38}$ We've performed our first SPG stimulator implantation for a cluster headache patient a few months ago with a good result. This technique involves placement of a needle at the ipsilateral SPG in the pterygopalatine fossa using a percutaneous infrazygomatic approach under fluoroscopic guidance. Of 5 patients with 18 acute cluster headaches over a period of 3 months, short-term (up to 1 hour) electric stimulation of the SPG completely aborted the pain in 11 attacks, partially aborted the pain in 3 attacks, and there was minimal/ no relief in 4 attacks. ${ }^{38}$ The mechanism of action of this therapy involves interruption of the postganglionic parasympathetic outflow and regulating the sensory inputs and processing in the nucleus caudalis of trigeminal. ${ }^{39}$ Following the beneficial effect of this therapy in patients with cluster headache, this modality has gained interest in treating patients with migrainous headache. ${ }^{39}$ However, the literature on SPG neuromodulation is mostly from small series of patients and needs to be validated by randomized clinical trials.

\section{Cervical spinal cord stimulation for craniofacial pain}

There are few studies evaluating the role of high cervical spinal cord stimulator for treating head or face pain. 4,40,41 The procedure is usually performed in a similar manner to a standard thoracic spinal cord stimulator with somatosensory evoked potential monitoring. The quadripolar paddle leads are implanted in a retrograde manner following a $\mathrm{C} 1$ hemilaminotomy with the proximal contacts directed at the cervicomedullary junction. A study evaluating the efficacy of cervical SCS in 41 patients with intractable upper limb and facial pain concluded that the patients with face pain did not respond to this therapy. ${ }^{4} \mathrm{~A}$ recent retrospective study ${ }^{41}$ showed that this therapy is a good option for trigeminal deafferentation pain with greater than $70 \%$ of patients in this group having a positive result, while not a good option for occipital neuralgic pain (only $28 \%$ response). However, this study is limited by its small sample size and thus large scale clinical trials are warranted. At Jefferson, we typically perform high cervical spinal cord stimulation for upper limb pain rather than headache or cervical pain.

\section{Trigeminal nucleus caudalis (TNC) dorsal root entry zone ablative procedures for complex craniofacial pain}

Nucleus caudalis dorsal root entry zone (DREZ) ablation has been shown to be effective in relieving refractory trigeminal neuropathic pain, atypical headache, complex craniofacial pain, anesthesia dolorosa, post-herpetic neuralgia, refractory pain associated with multiple sclerosis, brain stem infarction, and terminal cancers. The TNC is primarily associated with receiving and integrating nociceptive sensations, therefore lesioning at the node might interrupt the pain pathways and spontaneous pain generation in patients with deafferentation pain syndrome. Trigeminal tractotomy and nucleotomy (TR-NC) involves lesioning the descending spinal trigeminal tracts in the medulla along with the nucleus caudalis (typically performed under image guidance), whereas nucleus caudalis DREZ involves lesioning the whole substantia gelatinosa at the nucleus caudalis level (usually open surgery). Kanpolat et al reported significant pain relief in 19 or 21 patients with atypical facial pain following CT guided TR-NC.42 Bullard et $\mathrm{al}^{43}$ evaluated the efficacy of caudalis DREZ surgery for complex craniofacial pain and found excellent pain relief immediately with sustained (67\%) response rate at 1 year follow-up. Since caudalis DREZ lesion can be associated with significant life-threating surgical complications during manipulation of the brain stem, some studies have advocated TR-NC as a first-step procedure given the minimal invasiveness, low complication rate, and high efficacy associated with TR-NC. ${ }^{42,44}$ Recent improvement in surgical technique and employment of

Table 2. Prospective trials of PNS for migraine

\begin{tabular}{|l|c|c|l|}
\hline Author & aka & N & \multicolumn{1}{|c|}{ Result } \\
\hline Lipton et al, ${ }^{35} 2009$ & PRISM & 132 & no statistically significant reduction in headache days with ONS vs sham \\
\hline Saper et al, ${ }^{36} 2011$ & ONSTIM & 66 & $39 \%$ responder rate to adjustable ONS \\
\hline Silberstein et al, ${ }^{37} 2012$ & & 157 & reduction in headaches and MIDAS score with ONS \\
\hline
\end{tabular}


CT- guided placement of percutaneous radiofrequency electrode have made trigeminal tractotomy and nucleotomy a much safer procedure. Thompson et al reported a small but promising case series of intractable facial pain patients treated with percutaneous CT guided trigeminal nucleotomy under general anesthesia with good pain control at 6 months. We will be performing our first such procedure here at Jefferson in the upcoming weeks. ${ }^{45}$

Motor cortex stimulation (MCS) has been used for medically refractory syndromes such as post-stroke central pain, thalamic pain, pain secondary to traumatic brain injury, and atypical facial pain/ neuropathic facial pain. Though it was first reported $^{46}$ in 1993 with $60-90 \%$ relief in pain intensity for trigeminal neuropathic pain (TNP) the true efficacy is highly controversial and is now only considered as one of the last resorts in the management options. Monsalve ${ }^{47}$ evaluated the efficacy of MCS for facial chronic TNP in a systematic review and found that of 126 relevant studies and 118 patients, 100 $(84.7 \%)$ underwent permanent implantation and $84 \%$ of those had good pain relief. Raslan et al ${ }^{48}$ reported that 8 of 11 patients underwent permanent implantation and MCS for TNP and 5 continued to experience pain relief at a mean follow up of 33 months. In a prospective RCT for efficacy of MCS in 7 patients with chronic craniofacial pain syndromes, 6 of 7 reported significant pain relief. ${ }^{49} \mathrm{~A}$ larger prospectiverandomized trial for MCS is required to validate this modality as a primary therapy for patients with headache and facial pain; currently, we do not perform MCS at Jefferson.

\section{SUMMARY}

Complex craniofacial pain and refractory headaches can be challenging conditions to manage both medically and surgically. There are fairly well established neurosurgical managements of $\mathrm{TN}, \mathrm{a}$ very narrow spectrum of facial pains. On the other hand, due to complexity in the presentation and categorization of headaches/ facial pain in general, the best effective surgical management for majority of patients are unclear. With the technological advances and success of neurostimulation therapy in small trials, there is a resurgence of interest in the role of neurostimulation as well as ablative therapies. To date, there is a paucity of reliable evidence in the literature on the efficacy of neuromodulations for primary headache and craniofacial pain, which renders most of the above mentioned therapies non-FDA approved. Large scale prospective randomized controlled trials are needed to better understand the therapies that are most beneficial.

\section{REFERENCES}

1. Burchiel KJ. A new classification for facial pain. Neurosurgery 2003;53:1164-6; discussion 6-7.

2. Devor M, Govrin-Lippmann R, Rappaport $\mathrm{ZH}$. Mechanism of trigeminal neuralgia: an ultrastructural analysis of trigeminal root specimens obtained during microvascular decompression surgery. Journal of neurosurgery 2002;96:532-43.

3. Barker FG, 2nd, Jannetta PJ, Bissonette DJ, Larkins MV, Jho HD. The long-term outcome of microvascular decompression for trigeminal neuralgia. The New England journal of medicine 1996;334:1077-83.

4. Simpson BA, Bassett G, Davies K, Herbert C, Pierri M. Cervical spinal cord stimulation for pain: a report on 41 patients. Neuromodulation : journal of the International Neuromodulation Society 2003;6:20-6.

5. Miller JP, Magill ST, Acar F, Burchiel KJ. Predictors of long-term success after microvascular decompression for trigeminal neuralgia. Journal of neurosurgery 2009;110:620-6.

6. Gunther T, Gerganov VM, Stieglitz L Ludemann W, Samii A, Samii M. Microvascular decompression for trigeminal neuralgia in the elderly: long-term treatment outcome and comparison with younger patients. Neurosurgery 2009;65:477-82; discussion 82

7. Sekula RF, Jr., Frederickson AM, Jannetta PJ, Quigley MR, Aziz KM, Arnone GD. Microvascular decompression for elderly patients with trigeminal neuralgia: a prospective study and systematic review with meta-analysis. Journal of neurosurgery 2011;114:172-9.

8. Rughani Al, Dumont TM, Lin CT, Tranmer BI, Horgan MA. Safety of microvascular decompression for trigeminal neuralgia in the elderly. Clinical article. Journal of neurosurgery 2011;115:202-9.

9. Zhang H, Lei D, You C, Mao BY, Wu B, Fang Y. The long-term outcome predictors of pure microvascular decompression for primary trigeminal neuralgia. World neurosurgery 2013;79:756-62.
10. Garcia M, Naraghi R, Zumbrunn T, Rosch J, Hastreiter P, Dorfler A. High-resolution 3D-constructive interference in steadystate MR imaging and 3D time-of-flight MR angiography in neurovascular compression: a comparison between 3T and 1.5T. AJNR American journal of neuroradiology 2012;33:1251-6.

11. Thomas KL, Vilensky JA. The anatomy of vascular compression in trigeminal neuralgia. Clinical anatomy (New York, NY) 2014;27:89-93.

12. Revuelta-Gutierrez R, Martinez-Anda JJ, Coll JB, Campos-Romo A, Perez-Pena N. Efficacy and safety of root compression of trigeminal nerve for trigeminal neuralgia without evidence of vascular compression. World neurosurgery 2013;80:385-9.

13. North RB, Kidd DH, Piantadosi S, Carson BS. Percutaneous retrogasserian glycerol rhizotomy. Predictors of success and failure in treatment of trigeminal neuralgia. Journal of neurosurgery 1990;72:851-6.

14. Kondziolka D, Lunsford LD. Percutaneous retrogasserian glycerol rhizotomy for trigeminal neuralgia: technique and expectations. Neurosurgical focus 2005;18:E7.

15. Burchiel KJ. Percutaneous retrogasserian glycerol rhizolysis in the management of trigeminal neuralgia. Journal of neurosurgery 1988;69:361-6.

16. Pollock BE. Percutaneous retrogasserian glycerol rhizotomy for patients with idiopathic trigeminal neuralgia: a prospective analysis of factors related to pain relief. Journal of neurosurgery 2005;102:223-8.

17. Bender M, Pradilla G, Batra S, et al. Effectiveness of repeat glycerol rhizotomy in treating recurrent trigeminal neuralgia. Neurosurgery 2012;70:1125-33; discussion 33-4.

18. Harries AM, Mitchell RD. Percutaneous glycerol rhizotomy for trigeminal neuralgia: safety and efficacy of repeat procedures. British journal of neurosurgery 2011;25:268-72.

19. Missios S, Mohammadi AM, Barnett GH. Percutaneous treatments for trigeminal neuralgia. Neurosurgery clinics of North America 2014;25:751-62.

20. Son BC, Kim HS, Kim IS, Yang SH, Lee SW Percutaneous radiofrequency thermocoagulation under fluoroscopic image-guidance for idiopathic trigeminal neuralgia. Journal of Korean Neurosurgical Society 2011;50:446-52.

21. Kanpolat Y, Savas A, Bekar A, Berk C. Percutaneous controlled radiofrequency trigeminal rhizotomy for the treatment of idiopathic trigeminal neuralgia: 25-year experience with 1,600 patients. Neurosurgery 2001;48:524-32; discussion 32-4

22. Taha JM, Tew JM, Jr., Buncher CR. A prospective 15-year follow up of 154 consecutive patients with trigeminal neuralgia treated by percutaneous stereotactic radiofrequency thermal rhizotomy. Journal of neurosurgery 1995;83:989-93. 
23. Zakrzewska JM, Jassim S, Bulman JS. A prospective, longitudinal study on patients with trigeminal neuralgia who underwent radiofrequency thermocoagulation of the Gasserian ganglion. Pain 1999;79:51-8.

24. Fraioli B, Esposito V, Guidetti B, Cruccu G, Manfredi $M$. Treatment of trigeminal neuralgia by thermocoagulation, glycerolization, and percutaneous compression of the gasserian ganglion and/or retrogasserian rootlets: long-term results and therapeutic protocol. Neurosurgery 1989;24:239-45.

25. Xu SJ, Zhang WH, Chen T, Wu CY, Zhou MD. Neuronavigator-guided percutaneous radiofrequency thermocoagulation in the treatment of intractable trigeminal neuralgia. Chinese medical journal 2006;119:1528-35.

26. Regis J, Metellus $P$, Hayashi M, Roussel $P$ Donnet A, Bille-Turc F. Prospective controlled trial of gamma knife surgery for essential trigeminal neuralgia. Journal of neurosurgery 2006;104:913-24.

27. Pollock BE, Ecker RD. A prospective costeffectiveness study of trigeminal neuralgia surgery. Clin J Pain 2005;21:317-22.

28. Tatli M, Satici O, Kanpolat $Y$, Sindou M. Various surgical modalities for trigeminal neuralgia: literature study of respective long-term outcomes. Acta neurochirurgica 2008;150:243-55.

29. The International Classification of Headache Disorders: 2nd edition. Cephalalgia : an international journal of headache 2004;24 Suppl 1:9-160.

30. Wall PD, Sweet WH. Temporary abolition of pain in man. Science (New York, NY) 1967:155:108-9.

31. Weiner RL, Reed KL. Peripheral neurostimulation for control of intractable occipital neuralgia. Neuromodulation : journal of the International Neuromodulation Society 1999;2:217-21.
32. Slavin KV, Nersesyan H, Colpan ME, Munawar $\mathrm{N}$. Current algorithm for the surgical treatment of facial pain. Head $\&$ face medicine 2007:3:30.

33. Deogaonkar M, Slavin KV. Peripheral nerve/ field stimulation for neuropathic pain. Neurosurgery clinics of North America 2014;25:1-10.

34. Hann S, Sharan A. Dual occipital and supraorbital nerve stimulation for chronic migraine: a single-center experience, review of literature, and surgical considerations. Neurosurgical focus 2013;35: E9.

35. Lipton R GP, Cady R et al. PRISM study: occipital nerve stimulation for treatmentrefractory migraine. Cephalalgia 29(Suppl. 1),S30 (2009)

36. Saper JR, Dodick DW, Silberstein SD, McCarville S, Sun M, Goadsby PJ. Occipital nerve stimulation for the treatment of intractable chronic migraine headache: ONSTIM feasibility study. Cephalalgia: an international journal of headache 2011;31:271-85.

37. Silberstein SD, Dodick DW, Saper J, et al. Safety and efficacy of peripheral nerve stimulation of the occipital nerves for the management of chronic migraine: results from a randomized, multicenter, double-blinded, controlled study. Cephalalgia : an international journal of headache 2012;32:1165-79.

38. Ansarinia M, Rezai A, Tepper SJ, et al. Electrical stimulation of sphenopalatine ganglion for acute treatment of cluster headaches. Headache 2010;50:1164-74.

39. Khan S, Schoenen J, Ashina M. Sphenopalatine ganglion neuromodulation in migraine: what is the rationale? Cephalalgia: an international journal of headache 2014; 34:382-91.

40. Barolat G, Knobler RL, Lublin FD. Trigeminal neuralgia in a patient with multiple sclerosis treated with high cervical spinal cord stimulation. Case report. Applied neurophysiology 1988; 51:333-7.
41. Tomycz ND, Deibert CP, Moossy JJ. Cervicomedullary junction spinal cord stimulation for head and facial pain. Headache 2011:51:418-25

42. Kanpolat Y, Kahilogullari G, Ugur HC, Elhan $\mathrm{AH}$. Computed tomography-guided percutaneous trigeminal tractotomy-nucleotomy. Neurosurgery 2008;63:ONS147-53; discussion ONS53-5.

43. Bullard DE, Nashold BS, Jr. The caudalis DREZ for facial pain. Stereotactic and functional neurosurgery 1997;68:168-74.

44. Kanpolat Y, Savas A, Ugur HC, Bozkurt M. The trigeminal tract and nucleus procedures in treatment of atypical facial pain. Surgical neurology 2005;64 Suppl 2:S96100; discussion S-1.

45. Thompson EM, Burchiel KJ, Raslan AM. Percutaneous trigeminal tractotomy-nucleotomy with use of intraoperative computed tomography and general anesthesia: report of 2 cases. Neurosurgical focus 2013;35:E5

46. Meyerson BA, Lindblom $U$, Linderoth $B$, Lind G, Herregodts P. Motor cortex stimulation as treatment of trigeminal neuropathic pain. Acta neurochirurgica Supplementum 1993:58:150-3.

47. Monsalve GA. Motor cortex stimulation for facial chronic neuropathic pain: A review of the literature. Surgical neurology international 2012;3:S290-311.

48. Raslan AM, Nasseri M, Bahgat D, Abdu E, Burchiel KJ. Motor cortex stimulation for trigeminal neuropathic or deafferentation pain: an institutional case series experience. Stereotactic and functional neurosurgery 2011;89:83-8

49. Lefaucheur JP, Drouot $X$, Cunin $P$, et al. Motor cortex stimulation for the treatment of refractory peripheral neuropathic pain. Brain : a journal of neurology 2009;132:1463-71. 